\title{
Quantum State Measurement by Realistic Heterodyne Detection
}

\author{
Matteo G. A. Paris * \\ Arbeitsgruppe 'Nichtklassiche Strahlung' der Max-Planck-Gesellschaft an der Humboldt-Universitat zu Berlin, \\ Rudower Chaussee 5, 12489 Berlin, Germany ${ }^{\dagger}$
}

(4 Aug 1995)

The determination of the quantum properties of a single mode radiation field by heterodyne or double homodyne detection is studied. The realistic case of not fully efficient photodetectors is considered. It is shown that a large amount of quite precise information is avalaible whereas the completeness of such information is also discussed. Some examples are given and the special case of states expressed as a finite superposition of number states is considered in some detail.

1994 PACS number(s): 42.50.Dv; 03.65.Bz; 42.65.Ky.

\section{INTRODUCTION}

In order to get information about a quantum state one has to measure some observable. A question immediately arises: is this information complete? Namely, does it fully specify the quantum state under examination ? The answer is generally negative: the measurement of only one observable show up only an aspect of a physical system whereas a complete description requires a deeper inspection. However, the measurement of several different observables could require considerable efforts. Therefore it is a matter of interest to compare the simplicity and the feasibility of a measurement, or a set of measurements, with the provided amount of information. In addition, one has to weigh up the precision of such an information.

The complete description of a quantum state is contained in the density operator $\hat{\rho}$, or for pure state in the wave function. Therefore, a measurement leading to the density matrix in some representation provides, in principle, all the knowable information about the measured state. It has been shown theoretically [1] that the Wigner function $W_{0}(\alpha, \bar{\alpha})$ [2] of a field mode can be reconstructed starting from the homodyne measurements of a continuos set $\varphi \in[-\pi, \pi)$ of field quadratures $\hat{x}_{\varphi}=1 / 2\left(a e^{-i \varphi}+a^{\dagger} e^{i \varphi}\right)$. Later this procedure has been applied to coherent and squeezed states [3] using a finite set of phases $\varphi_{i}, i=1, \ldots 27$, upon a smoothing on experimental data. More recently a procedure suitable to finite sampling has been suggested for recovering matrix elements in the Fock representation [4] and latest

\footnotetext{
*E-mail Address: PARIS@PV.INFN.IT.

†Permanent Address: Dipartimento di Fisica 'Alessandro Volta', Universitá di Pavia, v. Bassi 6, 27100, Pavia, Italia.
}

developments have extended the method to data coming from inefficient detectors [5]. These various procedures, unitarily referred to as quantum tomography, provide a nice and powerful tools for investigating the quantum properties of radiation field leading to the maximum information achievable on the measured state. However, they require the detection of many field quadratures $\hat{x}_{\varphi_{j}}$, $j=, 1, \ldots, N$ and thus a lot of repeated measurements on the state under examination. A systematic approach to precision of quantum tomography is in progress [6] however, a preliminary study [7] has indicated that its determination of some field properties can be very noisy relative to the direct measurements of the same quantities.

\section{REALISTIC HETERODYNE DETECTION}

In this paper a different approach to quantum state measurement will be investigated. The crucial remark is that the density matrix in the coherent state representation $\langle\alpha|\hat{\rho}| \alpha\rangle$ is a positive definite function and thus can be directly measured for any quantum state of radiation field. Indeed, it is known that the so called Husimi $Q$-function $W_{-1}(\alpha, \bar{\alpha})=\langle\alpha|\hat{\rho}| \alpha\rangle$ represents the outcomes probability distribution 810 of both the heterodyne [11] and the double homodyne [12] detection scheme when equipped with ideal photodetectors. On the other hand its smoothed versions

$$
W_{s}(\alpha, \bar{\alpha})=\int \frac{d^{2} \beta}{\pi} W_{-1}(\beta, \bar{\beta}) \exp \left\{-2 \frac{|\alpha-\beta|^{2}}{1+s}\right\}
$$

emerge from realistic devices in which not fully efficient detectors are involved. The parameter $s$ depends on quantum efficiency as [13,14

$$
s=1-2 \eta^{-1}
$$

Starting from heterodyne, or equivalently from double homodyne [15], detection we can evaluate some quantity $O$ of interest as an average over the experimental distribution

$$
O=\langle\hat{O}\rangle=\int \frac{d^{2} \alpha}{\pi} W_{s}(\alpha, \bar{\alpha}) \mathcal{F}_{s}[\hat{O}](\alpha, \bar{\alpha}),
$$

where $\mathcal{F}_{s}[\hat{O}](\alpha, \bar{\alpha})$ is a non operatorial function related to the $s$-ordering, in the boson operator expansion, of the corresponding observable $\hat{O}[16]$. Denoting by $\{\hat{O}\}_{s}$ the $s$-ordered form of an operator we have for example 


$$
\begin{gathered}
a^{\dagger} a=\left\{a^{\dagger} a\right\}_{s}+\frac{1}{2}(s-1) \\
a^{\dagger 2} a^{2}=\left\{a^{\dagger 2} a^{2}\right\}_{s}+2(s-1)\left\{a^{\dagger} a\right\}_{s}+\frac{1}{2}(s-1)^{2},
\end{gathered}
$$

and thus

$$
\begin{gathered}
\mathcal{F}_{s}[\hat{n}](\alpha, \bar{\alpha})=|\alpha|^{2}+\frac{1}{2}(s-1) \\
\mathcal{F}_{s}\left[\widehat{n^{2}}\right](\alpha, \bar{\alpha})=|\alpha|^{4}+(2 s-1)|\alpha|^{2}+\frac{1}{2} s(s-1),
\end{gathered}
$$

for the mean photon number and for the number fluctuations. Similarly, we can investigate the squeezing properties of the examined state by means of the $s$-ordering of the field quadrature fluctuations

$$
\mathcal{F}_{s}\left[\widehat{x_{\varphi}^{2}}\right](\alpha, \bar{\alpha})=\frac{1}{4}\left[\alpha^{2} e^{-2 i \varphi}+\bar{\alpha}^{2} e^{2 i \varphi}+2|\alpha|^{2}+s\right],
$$

and also checking the uncertainty product. This procedure can be generalized in order to evaluate any field correlation which is of interest. In fact, any $t$-ordered moment $\left\{a^{\dagger n} a^{n+d}\right\}_{t}$, with arbitrary $t$, can be written in terms of a finite number of $s$-ordered moment by means of the formula 16]

$$
\begin{aligned}
& \left\{a^{\dagger n} a^{n+d}\right\}_{t}= \\
& \quad \sum_{k=0}^{n} \frac{(d+n) !}{(d+k) !}\left(\begin{array}{c}
n \\
k
\end{array}\right)\left(\frac{s-t}{2}\right)^{n-k}\left\{a^{\dagger n} a^{n+k}\right\}_{s},
\end{aligned}
$$

where also $s$ is arbitrary. The expectation value of any $t$ ordered moment can thus be evaluated by an average over the different ordered distribution $W_{s}(\alpha, \bar{\alpha})$. The special case in which the parameters $t$ and $s$ are choosen to be $t=1$ and $s=1-2 \eta^{-1}$ is of interest as it allows to obtain the normal ordered field correlations $\left\langle a^{\dagger n} a^{n+d}\right\rangle$ in terms of a finite numbers of heterodyne measured moments $\left\langle a^{\dagger k} a^{k+d}\right\rangle_{\eta}$. We have

$$
\begin{aligned}
\left\langle a^{\dagger n} a^{n+d}\right\rangle & =\sum_{k=0}^{n} \frac{(d+n) !}{(d+k) !}\left(\begin{array}{c}
n \\
k
\end{array}\right)\left(-\frac{1}{\eta}\right)^{n-k} \\
& \times \int \frac{d^{2} \alpha}{\pi} W_{1-2 \eta^{-1}}(\alpha, \bar{\alpha}) \alpha^{k+d} \bar{\alpha}^{k},
\end{aligned}
$$

or in a more compact form

$$
\begin{aligned}
\left\langle a^{\dagger n} a^{n+d}\right\rangle & =\frac{(-)^{n} n !}{\eta^{n}} \\
& \times \int \frac{d^{2} \alpha}{\pi} W_{1-2 \eta^{-1}}(\alpha, \bar{\alpha}) \alpha^{d} L_{n}^{d}\left(\eta|\alpha|^{2}\right)
\end{aligned}
$$

where $L_{n}^{d}(x)$ denotes Laguerre polynomials. An interesting application of Eq. (9) lies in the evaluation of the expectation value $\left\langle e^{i \hat{n} \phi}\right\rangle$ of the shift operator. Starting from normal ordering

$$
e^{i \hat{n} \phi}=\sum_{k}\left(e^{i \phi-1}\right)^{k} a^{\dagger k} a^{k} / k !
$$

we have, in fact

$$
\begin{aligned}
\left\langle e^{i \hat{n} \phi}\right\rangle= & \int \frac{d^{2} \alpha}{\pi} W_{1-2 \eta^{-1}}(\alpha, \bar{\alpha}) \\
& \times \quad \sum_{k=0}^{\infty}\left(\frac{1-e^{i \phi}}{\eta}\right)^{k} L_{k}\left(\eta|\alpha|^{2}\right) .
\end{aligned}
$$

Then, using properties of Laguerre polynomials [18], we arrive at the formula (valid for $0 \leq \phi<\arccos \left(1-\eta^{2} / 2\right)$ ]

$$
\begin{aligned}
\left\langle e^{i \hat{n} \phi}\right\rangle=\frac{\eta}{\eta-1+e^{i \phi}} & \int \frac{d^{2} \alpha}{\pi} W_{1-2 \eta^{-1}}(\alpha, \bar{\alpha}) \\
& \times \exp \left\{\frac{\eta\left(1-e^{i \phi}\right)|\alpha|^{2}}{1-e^{i \phi}-\eta}\right\} .
\end{aligned}
$$

Eq. (3) is also suitable for a reliable estimation of errors in the determination of the various expectation values. In practical situation, in fact, one has at disposal a finite sample of $W_{s}(\alpha, \bar{\alpha})$ and thus the integral in formula (3) has to replaced by the corresponding statistical sampling

$$
\bar{O}=\sum_{j \in \text { data }} W_{s}\left(\alpha_{j}, \bar{\alpha}_{j}\right) \mathcal{F}_{s}[\hat{O}]\left(\alpha_{j}, \bar{\alpha}_{j}\right) .
$$

According to the law of large numbers $\bar{O}$ approaches $\langle\hat{O}\rangle$ in the limit of infinite number of sampling data, whereas for finite sample we have a confidence interval $\bar{O} \pm \delta O$, with $\delta O$ given by

$$
\delta O=\sqrt{\sum_{j \in \text { data }} W_{s}\left(\alpha_{j}, \bar{\alpha}_{j}\right) \mathcal{F}_{s}^{2}[\hat{O}]\left(\alpha_{j}, \bar{\alpha}_{j}\right)-\bar{O}^{2}} .
$$

Some examples of the present reconstruction procedure can be given by means of numerical simulations of realistic heterodyne detection. In Fig. 1 I report the results for the mean photon number $\langle\hat{n}\rangle$ determination at different values of the quantum efficiency for coherent states of different amplitudes. Fig. 1 1 shows the results from heterodyne detection and Fig. 1 $1 \mathrm{~b}$ that ones from a direct photodetection. The two determinations are also compared in Fig. 1 c. In making such a comparison the same number of repeated measurements on the field have to be considered. In a scheme of $N$ repeated measurements of the quantity $x$, the accuracy $\delta x$ rescales as $\delta x \propto N^{-1 / 2}$. The proportionality costant generally depends on the kind of detection. If the outcomes $\bar{x}$ are distributed around the true value $x$ according to the probability $p(\bar{x} \mid x)$, the error for $N$ repeated measurements is always bounded by the Cramer-Rao inequality [19] $\delta x \geq(F N)^{-1 / 2}$, F being the Fisher information $F=\int d \bar{x}\left[\partial_{x} p(\bar{x} \mid x)\right]^{2} / p(\bar{x} \mid x)$. For Gaussian distributed data one has $F=1 / \sigma^{2}$, with $\sigma^{2}$ the variance of the distribution, and the lower bound for precision is achieved. In practical situations, in order to evaluate the precision $\delta x$, one takes advantage of the central limit theorem [19], which assures that the partial averages over a block of $N_{b}$ data is always Gaussian distributed around the global average over many blocks. 
Thus, one evaluates precision by dividing the ensemble of data into subensembles, and then calculates the r.m.s. deviation of subensemble averages with respect to the global one.

From Fig. 1 it is apparent that the method works also for low efficiency of the photodetectors even though the results are slightly more noisy than ones from direct detection. However, this level of introduced noise can be considered as admissible in sight of the further information available from the same data sample. Moreover, it has to be noticed (see Ref. [7]) that tomographic determination of $\langle\hat{n}\rangle$ introduces a very large amount of noise, even for unit quantum efficiency. Fig. 2 illustrates the application in recovering field fluctuations on a squeezed state and a number state for different values of the quantum efficiency.

About the determination of the phase some further considerations are in order. Heterodyne detection, in fact, is by itself a phase detectors as the marginal distribution

$$
P_{s}(\phi)=\int_{0}^{\infty} \rho d \rho W_{s}\left(\rho e^{i \phi}, \rho e^{-i \phi}\right)
$$

represents a phase distribution of the measured state [14,20. We have thus at disposal not only the mean value of the phase and the fluctuations but also the whole probability distribution. The distribution in Eq. (15) does not coincide (even for $\eta=1$ ) with the canonical phase distribution 10,20

$$
P(\phi)=\left\langle e^{i \phi}|\hat{\rho}| e^{i \phi}\right\rangle=\frac{1}{2 \pi} \sum_{n, m}^{\infty} e^{i(n-m) \phi} \rho_{n, m}
$$

and it is generally broadened relative to (16). In Fig. 3 the phase distribution obtained for a squeezed vacuum is reported for experiments carried out with different values of the quantum efficiency. The distributions broaden when the quantum efficiency decreases but the crucial information about phase bifurcation [21] is not lost also for for very inefficient detectors.

The results obtained until now can be summarized in the following assertions: i) starting from heterodyne detection many properties of the measured state can be determined at one go; ii) this determination is slightly more noisy than the corresponding ones from direct measurements of the same quantities one at times, even for unit quantum efficiency of the photodetectors. However, this behaviour is not unexpected as heterodyne detection involves the joint measurement of non commuting observables, and thus the unavoidably addition of noise by first principles [22,23]. This is not the case of quantum tomography where each homodyne measurement is independently performed and noise is introduced by data processing itself.

\section{DENSITY MATRIX RECONSTRUCTION}

Let us now deal with the completeness of the information coming from heterodyne detection. Is it possible, as an example, to determine the whole number distribution ? The matrix elements $\rho_{n+k, n}$ in the Fock representation are the expectation values of the generalized projectors

$$
\hat{P}_{n, n+k}=|n\rangle\left\langle n+k\left|=\frac{a^{\dagger n}}{\sqrt{n !}}\right| 0\right\rangle\langle 0| \frac{a^{n+d}}{\sqrt{(n+d) !}} .
$$

Using the Louisell expansion of the vacuum 17

$$
|0\rangle\langle 0|=\lim _{\varepsilon \rightarrow 1^{-}} \sum_{p} \frac{(-\varepsilon)^{p}}{p !} a^{\dagger p} a^{p}
$$

we can write $\hat{P}_{n, n+k}$ in terms of normal ordered moments

$$
\hat{P}_{n, n+k}=\frac{1}{\sqrt{n !(n+k) !}} \lim _{\varepsilon \rightarrow 1^{-}} \sum_{p} \frac{(-\varepsilon)^{p}}{p !} a^{\dagger n+p} a^{n+p+k}
$$

Eq. (19) is suitable to apply Eq. (7). After a straighforward calculation we get the reconstruction formula (3) for the matrix elements

$$
\begin{aligned}
\rho_{n+k, n} & =(-)^{n} \sqrt{\frac{n !}{(n+k) !}} \sum_{q=n}^{\infty}\left(\frac{1}{\eta}\right)^{q}\left(\begin{array}{c}
q \\
n
\end{array}\right) \\
& \times \int \frac{d^{2} \alpha}{\pi} W_{1-2 \eta^{-1}}(\alpha, \bar{\alpha}) \alpha^{k} L_{q}^{k}\left(\eta|\alpha|^{2}\right) .
\end{aligned}
$$

Unfortunately, Eq. (20) is not suitable for statistical sampling as the interchange of integration and summation is not mathematically allowed [24]. The analytical expression of $W_{s}(\alpha, \bar{\alpha})$ is needed and thus some smoothing procedure on sampled data is required, unavoidably introducing some a priori hypothesis on the state under examination [25]. However, Eq. (20) is far from being a purely formal tool. In many situations, in fact, one has some information about the considered state and thus some suggestions on parametryzing Wigner functions. Moreover, the distributions $W_{s}(\alpha, \bar{\alpha})$ for $s \leq-1$ are smoothed functions by themselves [16] and generally do not exhibit large or fast oscillations. Therefore we may expect the smoothing not leading to a dramatic lost of information and, at the same time, to not introduce fake information. The completeness of information coming from heterodyne detection cannot, anyhow, be claimed in a general way.

The reconstruction of the entire density matrix (in the Fock representation) and thus a complete description of the state is possible for the special case of states with a finite number of moments different from zero. Examples of such a states are provided by finite superpositions of number states 


$$
|\psi\rangle=\sum_{n=0}^{N} \psi_{n}|n\rangle .
$$

The latter can be produced in different manner in a high$\mathrm{Q}$ cavities [26,27] and also by a special non linear interaction [28]. If the moments $a^{\dagger n} a^{m}$ vanish for $n$ or $m$ beyond a certain value the series in Eq. (19) are actually truncated and Eq. (20) becomes suitable to a statistical sampling

$$
\begin{aligned}
\rho_{n+k, n} & =\frac{(-)^{n}}{\eta^{n}} \sqrt{\frac{n !}{(n+k) !}} \int \frac{d^{2} \alpha}{\pi} W_{1-2 \eta^{-1}}(\alpha, \bar{\alpha}) \alpha^{k} \\
& \times \sum_{p=0}^{N-n-k}\left(\begin{array}{c}
p+n \\
p
\end{array}\right)\left(\frac{1}{\eta}\right)^{p} L_{p+n}^{k}\left(\eta|\alpha|^{2}\right) .
\end{aligned}
$$

The value of $N$ has to be choosen large enough to ensure the cancellation of any moment $a^{\dagger N+j} a^{N+i}, i, j=0,1, \ldots$. In practice one can start with a large value of $N$ and then optimizing it by means of some stability criterion. In any case the precise value of $N$ is not needed by the algorithm. In Table I I report the results of this procedure when applied to the state

$$
|\psi\rangle=\frac{1}{\sqrt{2}}(|0\rangle+i|2\rangle),
$$

using photodetectors with an overall quantum efficiency equal to $\eta=0.9$. The reliability of the method is apparent. The same degree of precision can be obtained with lower efficiency using a larger sample.

The problem of reconstructing the density matrix of field-states with finite numbers occupation has been considered also by other authors, in particular in the context of the so-called Pauli's phase retrieval problem, where two experimentally determined probability distributions are needed. Orlowsky and Paul proposed in 29] an algorithm to recover the entire wavefunction (21), assuming that the moduli of the wavefunction are known in the position and momentum representation. Their method involves solving blocks of nonlinear equations after a decomposition of the wavefunction into Hermite polynomials. The resulting phase retrieval scheme is transparent, however it is recursive from above, namely it determines the coefficients $\psi_{n}$ from the highest index $N$ to the lowest. In addition, the value $N$ of the nonzero components of the wavefunction has to be known in advance. On the contrary, Eq. (22) allows recovering of the matrix elements $\rho_{n, m}$ one by one as an average over the experimental distribution and the value of $N$ is not needed by the algorithm. Bialynicka-Birula and Bialynicki-Birula in 30 considered the reconstruction problem starting from the knowledge of the photon number and phase (Pegg-Barnett) distributions. They suggested two different algorithms based on recursive iterations of Fast Fourier Transform from the number representation to the phase domain. Their algorithms work only for pure states whereas the present one can also be applied in the general case. In fact, the only requirement for writing Eq. (20) in the sampling-suited form (22) is that the measured state possesses only a finite number of moments different from zero. This condition can obviously be fulfilled also by finite mixtures. It has also to be mentioned that a detection scheme for the Pegg-Barnett phase distribution has not been devised yet. Thus the phase distribution needed by the algorithms in [30] can only be inferred by other state measurement schemes such as homodyning or quantum tomography.

Apart from the above considerations I consider the reliability of the present method in evaluating the confidence interval on matrix elements determinations as its main advantage.

\section{CONCLUSION}

In conclusion, quantum state measurement by heterodyne or double homodyne detectors has been shown to provide a large amount of quite precise information. It cannot lead to a complete specification of the measured state due to the singularity in some $s$-ordering $(s \leq-1)$ of operators. To the knowledge of the author it represents, at current time, the best compromise between the conflicting necessity of precise and complete state measurement.

I would thank 'Angelo Della Riccia' foundation for a research grant and Prof. Harry Paul for valuable hints. I am also very grateful to Valentina De Renzi for crucial encouragements.

[1] K. Vogel, H.Risken, Phys. Rev. A40, 2847 (1989).

[2] I adopt the usual definition for the generalized Wigner functions

$$
W_{s}(\alpha, \bar{\alpha})=\int \frac{d^{2} \lambda}{\pi} \operatorname{Tr}\left\{e^{\lambda a^{\dagger}+\bar{\lambda} a+\frac{1}{2} s|\lambda|^{2}}\right\} e^{\lambda \bar{\alpha}+\bar{\lambda} \alpha},
$$

in which the values $s=1,0,-1$ respectively correspond to Glauber $P$, symmetric Wigner and Husimi $Q$ functions.

[3] D. T. Smithey, M. Beck, M. G. Raymer, A. Faridani, Phys. Rev. Lett. 70, 1244 (1993).

[4] G. M. D'Ariano, C. Macchiavello, M. G. A. Paris, Phys. Rev. A50 4298 (1994).

[5] G. M. D'Ariano, U. Leonhardt, H. Paul, Phys. Rev. A52, R1801,(1995).

[6] G. M. D'Ariano, C. Macchiavello, M. G. A. Paris, N. Sterpi, unpubblished.

[7] G. M. D'Ariano, C. Macchiavello, M. G. A. Paris, Phys. Lett. A195, 31 (1994).

[8] U. Leonhardt, H. Paul, Phys. Rev. 47, 2460 (1993). 
[9] W. Schleich, A. Bandilla, H. Paul, Phys. Rev. A45, 6652 (1992); M. Freyberger, W. Vogel, W. Schleich, Quantum Opt. 5, 65 (1993); Phys. Lett. A176, 41 (1993).

[10] G. M. D'Ariano, M. G. A. Paris, Phys. Rev. 493022 (1994).

[11] J. H. Shapiro, S. S. Wagner, IEEE J. Quantum Electron. QE20, 803 (1984); H. P. Yuen, J. H. Shapiro, IEEE Trans. Inform. Theory IT26, 78 (1980).

[12] N.G. Walker, J.E. Carrol, Opt. Quantum Electr. 18, 355 (1986); N. G. Walker, J. Mod. Opt. 34, 15 (1987); Y. Lay, H. A. Haus, Quantum Opt. 1, 99 (1989); J. Noh, A. Fougeres, L. Mandel, Phys. Rev. Lett. 67, 1426 (1991); Phys. Rev. A45, 424 (1992).

[13] U. Leonhardt, H. Paul, Phys. Rev. 48, 4598 (1993).

[14] G. M. D'Ariano, C. Macchiavello, M. G. A. Paris, Phys. Lett. A198, 286 (1994).

[15] Throughout the paper any reference to the heterodyne detectors is also valid for the double homodyne detectors.

[16] K. E. Cahill, R. J. Glauber, Phys. Rev. 177, 1857 (1969); 177, 1882 (1969).

[17] W. H. Louisell, Quantum Statistical properties of Radiation, (Wiley, 1973).

[18] I. S. Gradshteyn, I. M. Ryzhik, Table of integral, series, and product, (Academic Press, 1980).

[19] H. Cramér, Mathematical Methods of Statistics, (Princeton Univ. Press, Princeton, NJ, 1946).

[20] U. Leonhardt, J. A. Vaccaro, B. Böhmer, H. Paul, Phys. Rev. A51, 84 (1995).

[21] W. Schleich, R. J. Horowicz, S. Varro, Phs. Rev. A40, 7405 (1989).

[22] H. P. Yuen, Phys. Lett. A91, 101, (1982).

[23] E. Arthurs, M. S. Goodman, Phys. Rev. Lett. 60, 2447 (1988).

[24] R. Baltin, J. Phys. A16, 2721 (1983).

[25] The needing of smoothing lies on the singularity of the antinormal ordering. The functions $\mathcal{F}_{s}\left[\hat{P}_{n, n+k}\right](\alpha, \bar{\alpha})$ have been obtained as a series of well-behaved functions however, actually they can be written in terms of the Dirac-delta distribution in the complex plane and its derivatives

$$
\mathcal{F}_{s}\left[\hat{P}_{n, n+k}\right](\alpha, \bar{\alpha})=\frac{1}{\sqrt{n !(n+k) !}} e^{|\alpha|^{2}} \frac{\partial^{2 n+k}}{\partial \alpha^{n+d} \partial \bar{\alpha}^{n}} \delta(\alpha) .
$$

[26] K. Vogel, V. M. Akulin, W. P. Schleich, Phys. Rev. Lett. 71, 1816 (1993).

[27] A. S. Parkins, P. Marte, P. Zoller, H. J. Kimble, Phys. Rev. Lett. 71, 3095, (1993).

[28] S. Ya. Kilin, D. B. Horoshko, Phys. Rev. Lett. 74, 5206 (1995).

[29] A. Orlowsky, H. Paul, Phys. Rev. A50, R921, (1994).

[30] Z. Bialynicka-Birula, I. Bialynicki-Birula, J. Mod. Opt. 41, 2203, (1994).
FIG. 1. Mean photon number determination by a simulated heterodyne and direct detections for different values of the quantum efficiency $\eta$. In (a) the results for three different coherent states of amplitude $\alpha=1$ (circle), $\alpha=2$ (square) and $\alpha=3$ (triangle) are reported for heterodyne detections. In (b) are reported the results from direct detections with the same number of repeated measurements on the same coherent states. The confidence intervals of both the determinations are evaluated as usual, by dividing the whole sample of $10^{5}$ data in subensembles and then calculating r.m.s. deviation with respect to the global average (see text). In (c) the accuracy of the two kinds of determination is compared. The noise (in $\mathrm{dB}$ ) added by heterodyne detection is shown as the ratio between the corresponding confidence intervals.

FIG. 2. Simulated determination of field fluctuations $\overline{\Delta x_{\varphi}^{2}}$ for $\varphi=0, \pi / 2$. Results for a squeezed state of total energy $\langle\hat{n}\rangle=1$ equally distributed between coherent amplitude and squeezing and a number state $\hat{\rho}=|1\rangle\langle 1|$ are reported. The upper and the lower curves are for the squeezed state ( $\varphi=0, \pi / 2$ respectively), the central one the result for number state (isotropic field distribution). Samples of $10^{5}$ data are used and the confidence intervals are evaluated as in Fig. 1.

FIG. 3. Phase distribution from heterodyne detection for a squeezed vacuum with squeezing photons $\langle\hat{n}\rangle=1$. The distributions are obtained with a sample of $10^{5}$ data for $\eta=0.25,0.5,0.75,1.0$. Broader distributions correspond to lower values of $\eta$. 
TABLE I. Reconstructed density matrix along with the confidence intervals for the state $|\psi\rangle=1 / \sqrt{2}(|0\rangle+i|2\rangle)$. The experiment has been simulated for quantum efficiency $\eta=0.9$ using a sample of $10^{6}$ data.

\begin{tabular}{cccc}
\hline \hline $.502 \pm .024$ & $(.004-i .003) \pm(.022+i .021)$ & $(.001-i .493) \pm(.037+i .038)$ & $\cdots$ \\
$(.004+i .002) \pm(.021+i .022)$ & $-.003 \pm .053$ & $(-.003+i .002) \pm(.018+i .018)$ & $\cdots$ \\
$(.001+i .493) \pm(.037-i .038)$ & $(-.003-i .002) \pm(.018+i .018)$ & $.500 \pm .031$ & $\cdots$ \\
$\cdots$ & $\cdots$ & $\cdots$ & $-.004 \pm .063$ \\
\hline \hline
\end{tabular}

